https://doi.org/10.15407/ujpe66.8.691

S. DILMI ${ }^{1}$ A. BOUMALI ${ }^{2}$

${ }^{1}$ University of El Oued, Fa. Exact Sciences, Lab. in Operator Theory and PDE

(39000, W. El-Oued, Algeria; e-mail: samia-dilmi@univ-eloued.dz)

${ }^{2}$ Laboratoire de Physique Appliquée et Théorique Université Larbi-Tébessi

(Tébessa, Algeria; e-mail: boumali.abdelmalek@gmail.com)

\title{
ESTIMATION OF ELECTRON \\ IMPACT IONIZATION RATES OF Li USING A NON-MAXWELLIAN DISTRIBUTION FUNCTION
}

\section{Introduction}

In dynamic systems, where ions are immediately exposed to higher electron temperatures, the electronimpact ionization (EII) could be of importance [1]. For this purpose, EII may be relevant for studies of solar flares, coronal nanoflare heating, supernova remnants, and merging clusters of galaxies [2,3]. EII can also have a considerable effect on the distribution of the state of charge for plasmas with a non-thermal electron power distribution. Within such plasmas, the high-energy tail of the distribution includes a large population of electrons residing above the EII rate.

In the process of impact electron ionization, an atom or ion can be stroke by an electron and ionizes, also giving up an electron:

$(Z, N)+\mathrm{e} \rightarrow(Z, N-1)+\mathrm{e}+\mathrm{e}$,

(C) S. DILMI, A. BOUMALI, 2021

ISSN 2071-0194. Ukr. J. Phys. 2021. Vol. 66, No. 8
$(Z, N)$ refers to an atom with atomic number $Z$ and $N$ electrons, so an electron collides with a atom (with a subsequent formation of a single ionized atom), or $(Z, N)$ refers to an ion with atomic number $Z$ and $N$ electrons, so an electron collides with a ion (with a subsequent formation of a doubly ionized atom).

In our case, an electron collides with a lithium atom (with a subsequent formation of a single-ionized lithium atom $\mathrm{Li}^{+}$). The ionization reaction considered in our work is as follows:

$\mathrm{Li}+\mathrm{e} \rightarrow \mathrm{Li}^{+}+\mathrm{e}+\mathrm{e}$.

Consequently, EII is important in the modeling of astrophysical systems, where these non-thermal distributions occur [4]. Knowing the position of hot electrons in plasmas is especially important due to their effect on the dynamics of plasma, the development of radiation, and energy balances [5]. EII is therefore appropriate for the analysis of astrophysical systems, 
where such non-thermal distributions occur [4]. Realizing the role of hot electrons in plasmas is incredibly significant because of their impact on the radiation production, energy balance, and plasma dynamics [5]. These electrons would cause considerable energy losses and have negative impacts on the stability and control over the plasma. In plasma physics and fusion, the non-Maxwellian and hot electrons turn out to be an interesting new topic to consider, because these electrons can play a significant role in the growth, evolution, and radiative properties of a broad range of plasma sources. The estimation of rates is based on the previous analytic concepts extracted from data sources, primarily Mihalas and Stone (1968) [6].

Such studies of hot electrons were tailored to specific experiments, and the results obtained were restricted to definite types of the energy distribution used to characterize the hot electrons. The relative insensitivity of the rates of collisional ionization, resonant excitation, and radiative recombination to the characteristic energy and functional shape of the electron distribution has significant consequences for collisional radiative two-temperature models. These usually involve the distributions of hot electrons whose characteristic energies are much greater than the largest transition of interest, and the sensitivity of the effects of hot electrons for the Gaussian and Maxwellian distributions was noted and observed separately [7].

This work is a theoretical and numerical study of the ionization rates of plasma with a population of electrons whose velocity distribution function is initially non-Maxwellian. The effects of the simultaneous presence of electrons with low energy and electrons with high energy on the calculation of ionization rates, as well as the energy distribution functions of electrons, are discussed.

The goal of this approach is to present the influence of hot electrons and to research the impact of the electron energy distribution functions on the measurement of the electron impact ionization rate of Li using a non-Maxwellian energy distribution in the case of low fractions of hot electrons. We will study the use of a non-Maxwellian distribution because of the results are satisfactory for low fractions of hot-electrons. We can see a very strong agreement between the two curves of ionization rates by using the power-law and the Gaussian electron distribution functions, respectively. The interval for the fraction of hot electrons is taken to be $[1-10] \%$.

The point study with the comparison of neutral helium ionization rates calculated with the non-Maxwellian power distribution functions of electrons [8] exhibits a great improvement of the curves, particularly for the Gaussian and power-law distribution functions.

\section{Theoretical Context}

\subsection{Ionization cross-section}

Ionization rates for different ions in both laboratory and astrophysical plasmas are critical for understanding the ionization balance. Many statistical formulae have been recommended for measuring the ionization rates and cross-sections.

As for the empiric formula for ionization crosssections, the Lotz formula has been frequently used in plasma-related fields [9]. Lotz [10, 14] ascertained a cross-section obtained from experimental measurements of weakly ionized ions of comparatively low- $Z$ elements:

$\sigma=\sum_{j=1}^{\mathrm{Nsc}} a_{j} \tau_{j} \frac{\ln u_{j}}{u_{j} x_{j}^{2}}\left(1-b_{j} \exp \left[-c_{j}\left(u_{j}-1\right)\right]\right)$,

where: $u_{j}=\frac{\varepsilon}{x_{j}}, \varepsilon(\mathrm{eV})$ is the energy of impact electron, $x_{j}(\mathrm{eV})$ is the binding energy of an electron in the $j$-th subshell, $\tau_{j}$ is the number of equivalent electrons of the $j$-th subshell, and $a_{j}, b_{j}$, and $c_{j}$ are the individual constants to be adjusted from the experimental data and some scaling.

For the ionization cross-section, Arnaud and Rothenflug [11] also used the following parametric formula, initially introduced by Younger $[12,13]$ :

$$
\begin{aligned}
& \sigma=\sum_{j} \frac{1}{u_{j} x_{j}^{2}}\left[a_{j}\left(1-\frac{1}{u_{j}}\right)+b_{j}\left(1-\frac{1}{u_{j}}\right)^{2}+\right. \\
& \left.+c_{j} \ln \left(u_{j}\right)+d_{j} \frac{\ln \left(u_{j}\right)}{u_{j}}\right] .
\end{aligned}
$$

The coefficients $a_{j}, b_{j}, c_{j}$, and $d_{j}$ are given in Table [9] together with the values of $x_{j}$. Using the Lotz $[10,14]$ and Arnaud et al. [11] formulae, we present the findings of empiric formula for electron impact ionization cross-section of Li. The findings obtained are illustrated in Fig. 1.

ISSN 2071-0194. Ukr. J. Phys. 2021. Vol. 66, No. 8 
Figure 1 presents the electron impact ionization cross-section for $\mathrm{Li}$ obtained by the Lotz and Arnaud et al. formulae, with a comparison with the results of experimental measurements which were carried out by Lotz [14]. The curves have maxima at approximately $20 \mathrm{eV}$. For the energy value $16.76 \mathrm{eV}$, the cross-sections calculated using the Lotz and Arnaud et al. formulae reach the values $4.32 \times 10^{16} \mathrm{~cm}^{2}$ and $9.97 \times 10^{16} \mathrm{~cm}^{2}$, respectively. At the same energy value $(16.76 \mathrm{eV})$, the electron impact ionization cross-section for $\mathrm{Li}$ reaches a maximum value of about $5.21 \times 10^{16} \mathrm{~cm}^{2}$ in the study of the experimental results presented in the work by Lotz [14]. This is in good agreement with the value $4.32 \times 10^{16} \mathrm{~cm}^{2}$ calculated by the Lotz formula in our work.

Due to various available neutral targets (atoms, molecules, radicals, clusters) and the considerable theoretical and experimental complexities, the issue involving the quantitative awareness of the absolute electron effect ionization cross-sections remains unsatisfactory $[15,16]$. Concerning the electron impact ionization of neutral particles, a considerable amount of experimental and theoretical researches in this century has been applied to the estimation of appropriate electron impact ionization cross-section functions. Though the remarkable efforts have been made, no full theoretical results were achieved so far [16].

The calculation codes are mostly designed for the calculation of the atomic structure and some atomic data related to atomic processes (recombination, ionization, excitation ...). They use theories or approximations that may be different for the calculation of atomic structure (Ref. [18] gives a summary of the most used codes).

Through the electron impact no resonant photo ionization and radiative recombination, auto ionization, and dielectronic recombination, FAC (Flexible Atomic Code) $[18,19]$ is usually prepared to accommodate the radiative transformation, direct collisional excitation, and ionization. The purpose of developing such a robust package is to combine different atomic processes into a common theoretical system and to provide a uniform flexible and easyto-use user interface for accessing all computational tasks. The FAC code would be used to calculate the cross-section of ionization by the electron impact using the relativistic approximation "Distorted

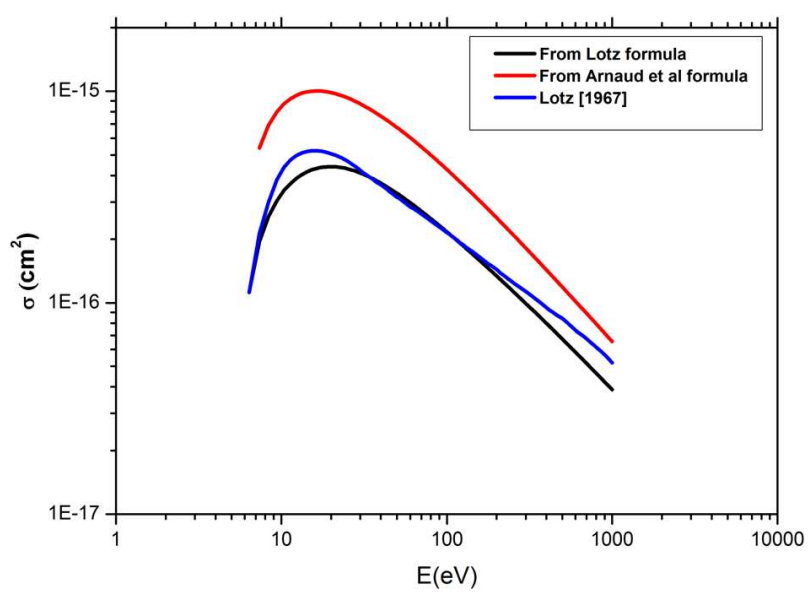

Fig. 1. Electron impact ionization cross-section for $\mathrm{Li}$ obtained by the Lotz and Arnaud et al. formulae

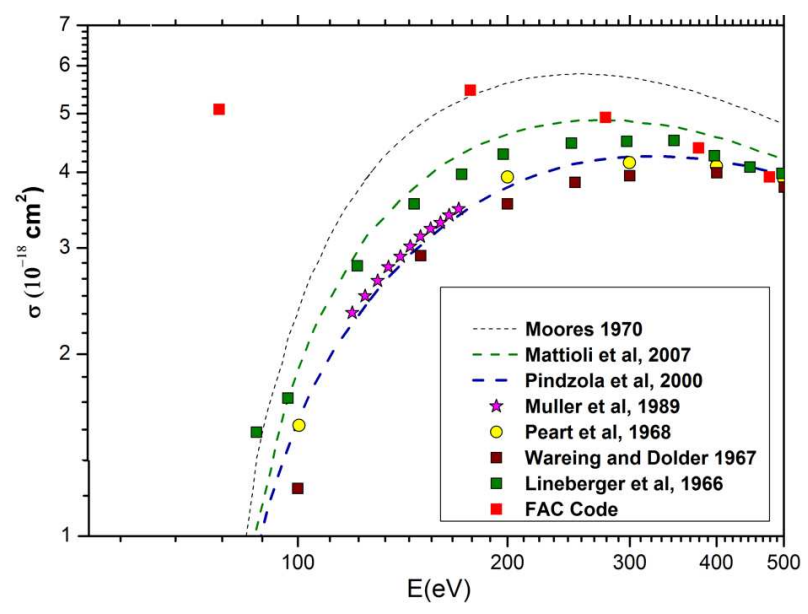

Fig. 2. Electron impact ionization cross-section for $\mathrm{Li}$ obtained by the FAC code

Wave method" (DW) and using the interpolationfactorization method $[18,19]$.

The electron impact ionization cross-section for $\mathrm{Li}$ was obtained using the FAC code. Figure 2 shows the results obtained by the $\mathrm{FAC}$ code in the energy interval from threshold to $500 \mathrm{eV}$.

The experimental data are taken from works [2027]. There are good agreements between the curves in Fig. 2. It comprises the results of different theoretical approaches, the distorted-wave-with exchange (DWX) approximation, the R-matrix method with pseudo states (RMPS), and the time-dependent closecoupling (TDCC) approach which all agree with one another. The curve representing the theoretical re- 
sults by Pindzola et al. [22] in Fig. 2 has been obtained by fitting a parametrized formula previously suggested by Younger $[12,13]$ to the DWX crosssections by Pindzola et al. [22].

For the further comparison Fig. 2 includes the recommended cross-sections proposed by Mattioli et al. [21] in the support of applications to controlled thermonuclear fusion and astrophysics. The calculation by Pindzola et al. [22] is up to $20 \%$ above the recommendation of Mattioli et al. which is mainly based on the available experimental cross-sections. The pioneering Coulomb-Born calculation carried out by Moores and Nussbaumer [20] yielded even larger cross-sections. Obviously, the agreement between theory and experiment is not totally satisfactory [27].

From the Table, we can notice a significant agreement between theoretical calculations with the FAC code and a few experiments data. The FAC code is used to calculate the electron impact ionization cross section using the DW relativistic approximation that gives satisfactory results for atoms and ions. This method involves the potential as felt by the incident electron due to the nucleus of the target ion and the electronic procession.

\subsection{Distribution Functions}

The role of the electron energy distribution is a subject of high importance in plasma physics, fusion, and astrophysics, as it plays a significant role in the development, creation, and radiative properties of a diverse variety of plasma sources. Consequently, the collisional-radiative atomic models affecting the effect of non-Maxwellian distributions and hot electrons are of extreme importance in atomic physics and for the

The different values of the electron impact ionization cross-section for Li obtained by the FAC code and the experimental data

\begin{tabular}{|c|c|c|c|c|}
\hline \multirow{2}{*}{$E(\mathrm{eV})$} & $\begin{array}{c}\sigma \\
\left(10^{-18} \mathrm{~cm}^{2}\right)\end{array}$ & $\begin{array}{c}\sigma \\
\left(10^{-18} \mathrm{~cm}^{2}\right)\end{array}$ & $\begin{array}{c}\sigma \\
\left(10^{-18} \mathrm{~cm}^{2}\right)\end{array}$ & $\begin{array}{c}\sigma \\
\left(10^{-18} \mathrm{~cm}^{2}\right)\end{array}$ \\
\cline { 2 - 5 } & $\begin{array}{c}\text { FAC } \\
\text { Code }\end{array}$ & $\begin{array}{c}\text { Moores } \\
{[20]}\end{array}$ & $\begin{array}{c}\text { Pindzola } \\
\text { et al. }[22]\end{array}$ & $\begin{array}{c}\text { Mattioli } \\
\text { et al. }[21]\end{array}$ \\
\hline 176 & 5.4 & 5.35 & - & - \\
279 & 4.9 & - & 4.9 & 4.9 \\
377 & 4.3 & - & 4.2 & 4.6 \\
480 & 3.9 & - & 4.0 & 4.2 \\
\hline
\end{tabular}

spectroscopic equipment that can establish the existence and characteristics of the distribution mechanism of electrons in plasmas [28].

Specific experiments and researches on hot electrons have contributed to the fixation of certain types of the distribution function of energy. Those types are represented in the following expressions [28]:

Maxwellian:

$F_{\mathrm{M}}\left(\varepsilon, T_{e}\right)=2 \sqrt{\frac{\varepsilon}{\pi T_{e}^{3}}} \exp \left[\frac{-\varepsilon}{T_{e}}\right]$

Gaussian:

$F_{\mathrm{G}}\left(\varepsilon, T_{e}\right)=\frac{1}{T_{e} \sqrt{\pi}}\left(\frac{2}{1+\operatorname{erf}\left(\frac{+\varepsilon_{0}}{T_{e}}\right)}\right) \exp -\left[\frac{\left(\varepsilon-\varepsilon_{0}\right)}{T_{e}}\right]^{2} ;$

Power-law:

$F_{\mathrm{p}}\left(\varepsilon, T_{e}\right)=\left(\frac{\gamma-1}{T_{e}^{1-\gamma}}\right) \varepsilon^{-\gamma}, \quad \varepsilon \geq T_{e}$

where $T_{e}, \varepsilon, \varepsilon_{0}$ are the energies of electrons corresponding to each distribution, and $\gamma$ is decay constant.

We use an energy distribution for estimating the ionization rates from the cross-sections. This helps one to observe the effects of the distribution functions of energy on the calculation of ionization rates.

\subsection{Non-Maxwellian ionization rates}

Within plasma, free electrons are represented by a certain energy distribution. The ionization rates are calculated by integrating a cross-section of an energydependent collision over the distribution functions of energy. The impressive quantity is the rate of ionization by the electron impact which is collected by averaging the product of the velocity of the electron by the ionization cross-section.

If the electron receives a sufficient energy (through super-elastic collisions), which is equivalent to neutral atom's ionizing potential, an ionizing collision will result in an electron impact process resulting in a lowenergy electron $\left(\varepsilon=\varepsilon_{1}+\varepsilon_{2}+E_{\text {ion }}\right)$ and an atomic ion. For the direct ionization, the ionization rates are defined by [28]:

$\tau=\int \mathrm{v} \sigma(\varepsilon) F(\varepsilon) d \varepsilon\left(\mathrm{cm}^{3} \mathrm{~s}^{-1}\right)$,

ISSN 2071-0194. Ukr. J. Phys. 2021. Vol. 66, No. 8 
where $v$ is the velocity of the incident electron, $\sigma(\varepsilon)$ is the impact ionization cross- sections calculated by the FAC code, $F(\varepsilon)$ is the electron energy distribution function, and $\varepsilon$ is the energy of the incident electron.

For single-temperature plasmas with the Maxwellian distribution of electron energy, the de-excitation and recombination rates could be calculated by the precise balance explicitly from the collisional excitation and ionization rates. In plasmas that have atoms in non-Maxwellian distributions, the crosssections of those inverse rates must be integrated throughout the entire distribution of electron energy. The associated rates could be expressed by a simple analytic formula by using the appropriate approximation for the energy dependence of the crosssections.

We extended our calculations to the case of plasma characterized by a non-Maxwellian electron energy distribution. This distribution could be used to model plasmas containing a small fraction of a few percent of hot electrons, sometimes found in the Universe or in the laboratory.

Induced plasmas often exhibit non-Maxwellian distribution functions for electrons that can be represented by two temperatures corresponding to the hot and cold populations. To calculate the ionization rates from cross-sections, we use a non-Maxwellian energy distribution function $F(\varepsilon)$. We have used the following non-Maxwellian model to evaluate the influence of electron energy distribution functions on the lithium ionization rate calculated in $[28,29]$ :

$F(\varepsilon)=\left(1-f_{\text {hot }}\right) F_{\mathrm{M}}\left(\varepsilon, T_{\text {bulk }}\right)+f_{\text {hot }} F_{X}\left(\varepsilon, T_{\text {hot }}\right)$,

where $f_{\text {hot }}$ is the normalized hot electron fraction, $F_{\mathrm{M}}\left(\varepsilon, T_{e}\right)$ is the Maxwell energy distribution function, and $F_{X}\left(\varepsilon, \varepsilon_{0}\right)$ is the electron energy distribution function. The order of magnitude of $T_{\text {bulk }}$ in our case is $1 \mathrm{eV}$, while $T_{\text {hot }}$ is $100 \mathrm{eV}$ and more. The exact value of $T_{\text {bulk }}$ does not affect the shape of the ionization rate curve. The fraction of cold electrons is very low, because we have chosen to study the high fraction of hot electrons.

Substituting Eq. (9) into (8) and taking the electron energy distribution function $F_{X}\left(\varepsilon, T_{e}\right)$ to be Maxwellian (Eq. (5)), Gaussian (Eq. (6)) and powerlaw (Eq. (7)) separately enabled us to obtain the ionization rates for various fractions of hot electrons $f_{\text {hot }}$.

\section{Results and Discussion}

Figures 4 and 5 display the results of measurements of the electron impact ionization rates for Li for the Maxwellian and Gaussian energy distribution functions for various fractions of hot electrons. The ionization rates for $\mathrm{Li}^{+}$are calculated from relation (8) by substituting formula (9) into (8) and replacing $F_{X}\left(\varepsilon, T_{e}\right)$ by the Maxwellian distribution function $F_{\mathrm{M}}\left(\varepsilon, T_{e}\right)($ Eq. $(5))$ for Fig. 4 and the Gaussian distribution function $F_{\mathrm{G}}\left(\varepsilon, T_{e}\right)$ (Eq. (6)) for Fig. 5 and this for different fractions of hot electrons $f_{\text {hot }}$. However, in Figs. 3 and 4, the rates increase very rapidly at low temperatures. In the case of high temperatures,

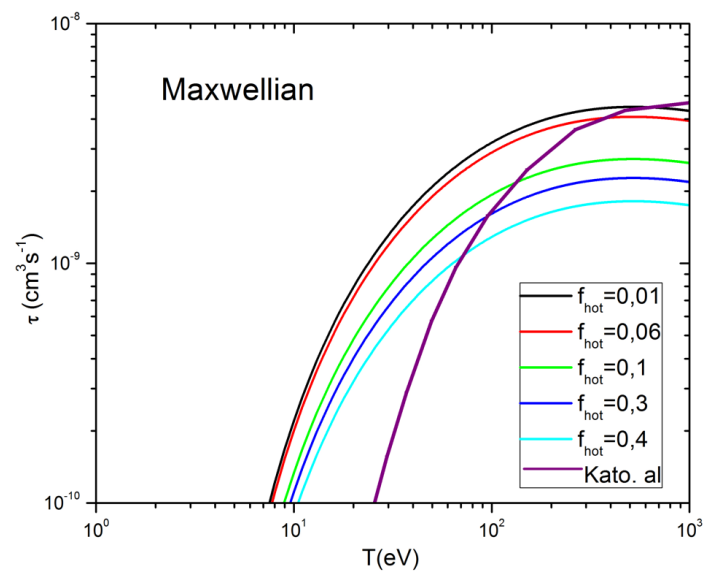

Fig. 3. The electron impact ionization rates for Li obtained using the Maxwellian electron energy distribution function and with regard for the effect of hot electrons, $f_{\text {hot }}$

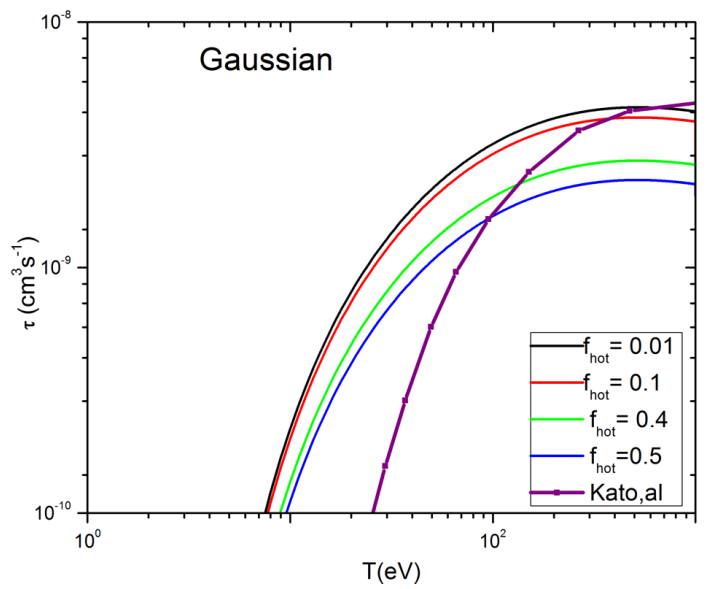

Fig. 4. The electron impact ionization rates for $\mathrm{Li}$ obtained using the Gaussian electron energy distribution function and the effects of hot electrons 

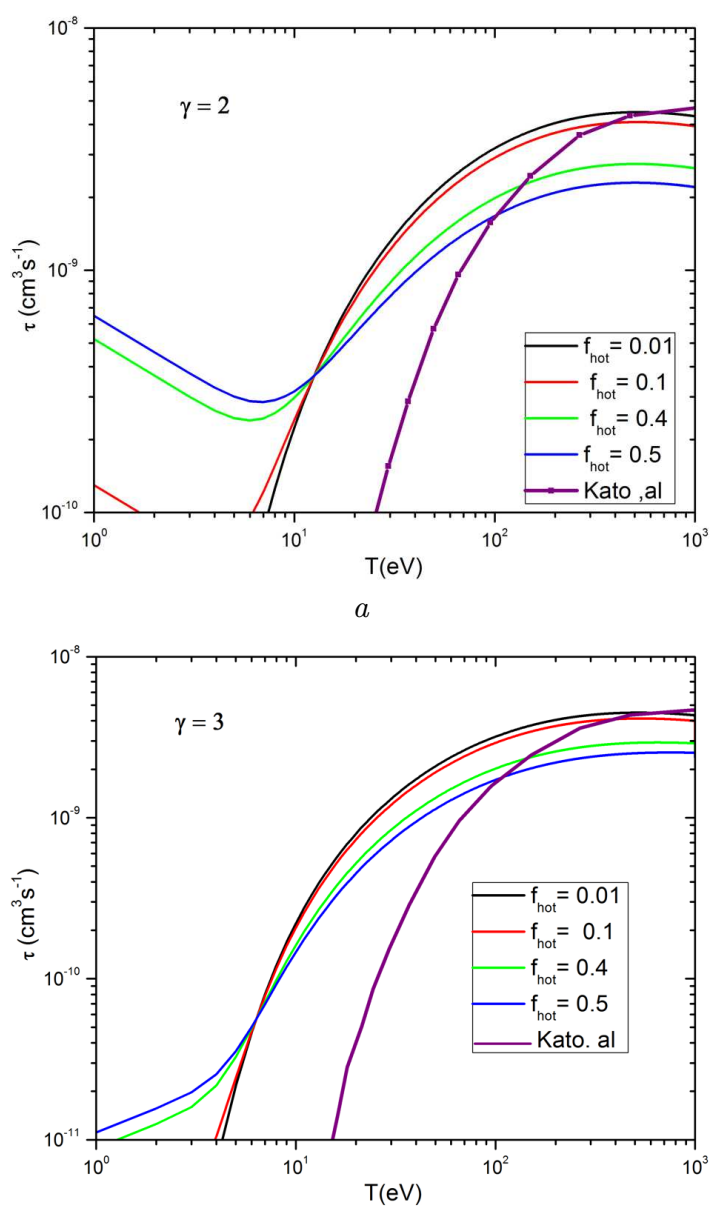

$b$

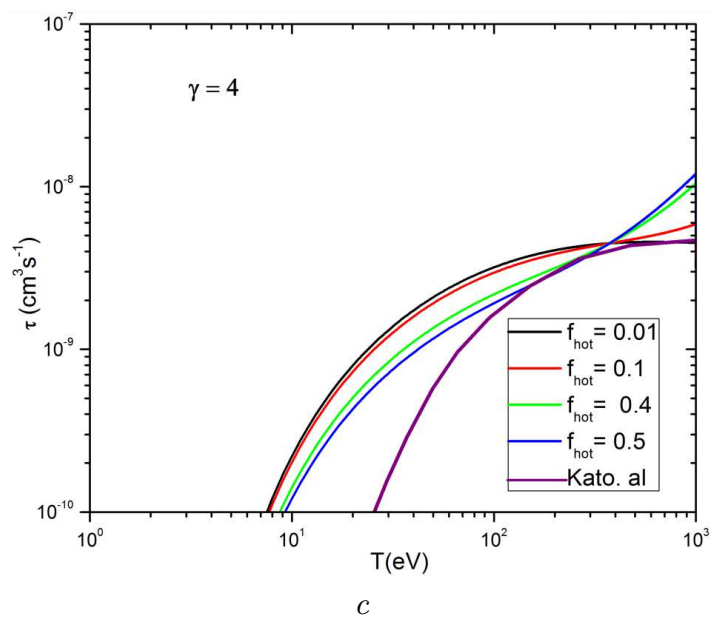

Fig. 5. The electron impact ionization rates for Li obtained using the power-law electron energy distribution function in for various decay constants: $\gamma=2(a), \gamma=3(b), \gamma=4(c)$ with regatd for the effects of hot electrons
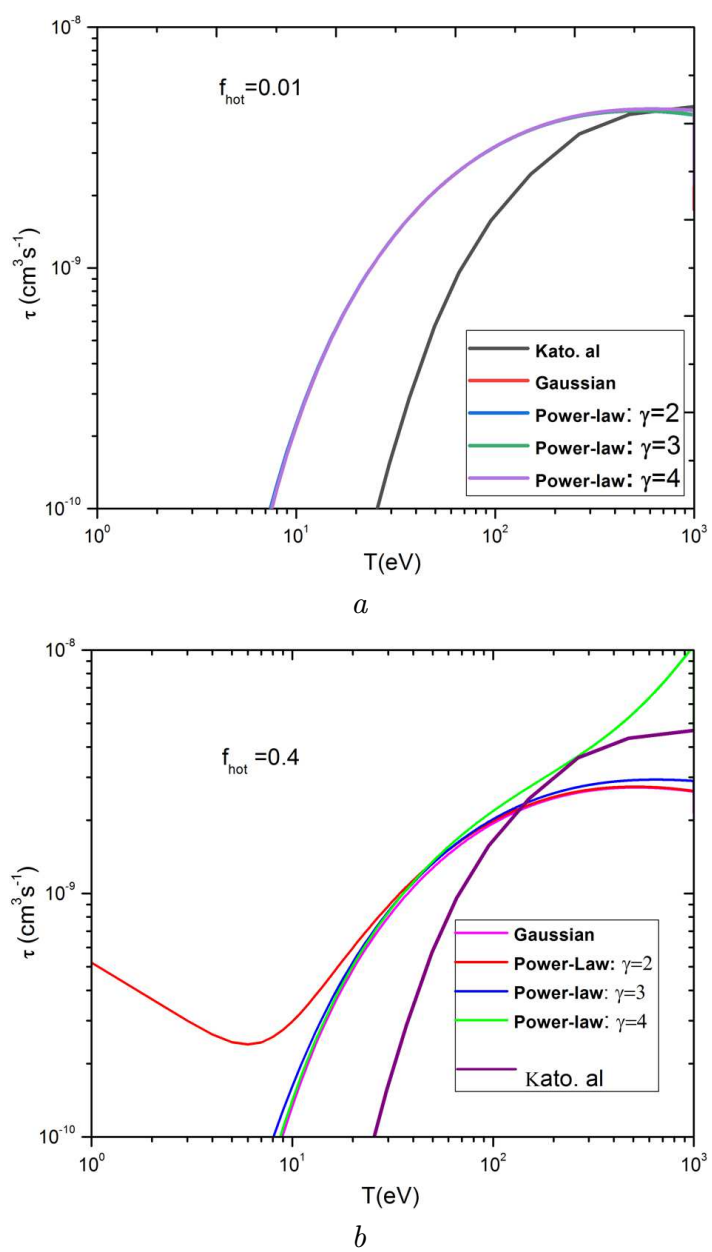

Fig. 6. The electron impact ionization rates for $\mathrm{Li}$ obtained using the Gaussian and power-law electron energy distribution functions for various decay constants $\gamma$ and for the fractions of hot electrons $f_{\text {hot }}=0.01(a)$ and $f_{\text {hot }}=0.4(b)$

it is noticed that the ionization rate curves are very sensitive to the fraction of hot electrons. We can see that the curves are gradually moving away from each other as the fraction of hot electrons increases.

The results obtained for the calculation of electron impact ionization rates for $\mathrm{Li}$ are compared with those published in [9]. In general, the acceptable agreement was noticed for the curves of Figs. 3 and 4. Significant deviations are observed for low temperatures in the energy range $20-200 \mathrm{eV}$. Indeed, at low temperatures ( $T$ less than $25 \mathrm{eV}$ ), the ionization rates are very sensitive to the behavior of the crosssections. There are considerable differences between the different theoretical and/or empiric methods.

ISSN 2071-0194. Ukr. J. Phys. 2021. Vol. 66, No. 8 
Born's approximation of the plane wave is not valid at low energies and yields cross-sections smaller than those obtained by a calculation based on the distorted wave method [9]. These deviations are acceptable in the interval $150-700 \mathrm{eV}$, while they are very small from $1000 \mathrm{eV}$. We found relative deviations of around $20 \%$.

Figure 5 shows the rate of electron impact ionization of Li for a power-law electron energy distribution function for different values of the decay constant $\gamma$.

In Fig. 5, $a$ and b, at low temperatures, the curves indicate the susceptibility to the fraction of hot electrons, as well as an increase in the constant of decay $\gamma$.

Starting from $500 \mathrm{eV}$ in Fig. 5, c, we notice the permissible variations in the range $10-100 \mathrm{eV}$. It is important to note that, particularly for the power-law distribution function, there is a considerable improvement in curves to the value of $\gamma=4$.

In Fig. 6, we can observe a very good agreement between the two ionization rate curves using the powerlaw and Gaussian distribution functions for the whole interval of energy and the fractions of hot electrons $[1-10] \%$. We also note that the value of $\gamma$ does not affect the estimate of calculations at a fixed value of the fraction of hot electrons [8]. The relative susceptibility of the ionization rate to the shape of the electron energy distribution functions, when $\gamma=2$ and the hot electron fraction assumes high values, has significant consequences for collisional radiative models at two temperatures. We note that when the temperature of electrons increases, the hot electron distribution takes a supra thermal form (non-Maxwellian) which has a long tail compared to the Maxwellian distribution at the thermal equilibrium.

\section{Conclusions}

For the determination of cross-sections, we have used the Lotz and Arnaud et al. formulae. The FAC code (Flexible Atomic Code) is introduced to calculate the electron impact ionization cross-section for $\mathrm{Li}$. We note that there is a significant agreement between theoretical calculations with the FAC code and a few experiments data. We choose a non-Maxwellian distribution function of energy to estimate the rates of ionization from cross-sections. First, it is shown that most collisional rates are much more sensitive to the fraction of hot electrons than to the exact functional form of the electron energy distributions. The higher the temperature, the larger the number of supra thermal electrons, which allows larger fractions of hot electrons to exhibit profound impacts. The ionization rates are very sensitive to the behavior of crosssections. There are considerable differences between the different theoretical and/or empiric methods. The use of non-Maxwellian energy distributions demonstrated the sensitivity of these rates to the electron energy distribution shape. The results obtained in the calculation of the electron impact ionization rates for $\mathrm{Li}$ using the different distribution functions of energy are compared with those published in [9]. In general, an acceptable agreement was noticed for the curves in various figures. Significant deviations are observed for low temperatures in the energy interval 20-200 eV.We have shown the exceptional sensitivity of the ionization rate for $\mathrm{Li}^{+}$taking the fraction of hot electrons into consideration.

1. D. Salzman. Atomic Physics in Hot Plasmas (Oxford University Press, 1998).

2. A.S. Shlyaptseva, S.B. Hansen, V.L. Kantsyrev, D.A. Fedin, N. Ouart, K.B. Fournier, U.I. Safronova. Advanced spectroscopic analysis of $0.8-1.0-$ MA Mo $x$ pinches and the influence of plasma electron beams on L-shell spectra of Mo ions. Phys. Rev. E67, 026409 (2003).

3. J. Colgan, H.L. Zhang, C.J. Fontes. Electron-impact excitation and ionization cross-sections for the $\mathrm{Si}, \mathrm{Cl}$, and $\mathrm{Ar}$ isonuclear sequences. Phys. Rev. A 77, 062704 (2008).

4. M. Davoudabadi, J.S. Shrimpton, F. Mashayek. On accuracy and performance of high-order finite volume methods in local mean energy model of non-thermal plasmas. J. Comp. Phys. 228, 2468 (2009).

5. R. Bartiromo, F. Bombarda, R. Giannella. Spectroscopic study of nonthermal plasmas. Phys. Rev. A 32, 531 (1985).

6. D. Mihalas, M E. Stone. Statistical equilibrium model atmospheres for early-type stars. III. Hydrogen and helium continua. Astrophys. J. 151, 293 (1968).

7. M.A. Mahmoud, Kh.A. Hamam. Studies of electron energy distribution function (EEDF) in lithium vapor excitation at $2 \mathrm{~S} \rightarrow 3 \mathrm{D}$ two-photon resonance. O. P. J 4, 195 (2014).

8. S. Dilmi, A. Boumali. Influence of the electron energy distribution function on the calculation of ionization rate in hot plasma. U.P.B. Sci. Bull. Series A 79, 249 (2017).

9. T. Kato, E. Asano. Comparison of recombination rate coefficients given by empirical formulas for ions from hydrogen through nickel NIFS-Data-Series 14, (1999).

10. W. Lotz. Electron-impact ionization cross-sections and ionization rate coefficients for atoms and ions from hydrogen to calcium. Zeitschr. f. Physik. 216, 241 (1968).

11. M. Arnaud, R. Rothenflug. An updated evaluation of recombination and ionization rates. Astron. Astrophys. Supp. Ser. 60, 425 (1985). 
12. S.M. Younger. Electron impact ionization rate coefficients and cross-sections for highly ionized iron. J. Quant. Spectrosc. Radiat. Transfer. 27, 541 (1982).

13. S.M. Younger. Electron-impact ionization cross-sections for highly ionized hydrogen- and lithium-like atoms. Phys. Rev. A 22, 111 (1980).

14. W. Lotz. Electron-impact ionization cross-sections and ionization rate coefficients for atoms and ions. Astrophys. J. Supp. 14, 207 (1967).

15. T.D. Mark, G.H. Dunn. Electron Impact Ionization (Springer, 1985).

16. R.S. Freund, L.C. Pitchford, B.V. McKay, A. Chutjian, S. Trajmar. Swarm Studies and Inelastic Electron Molecule Collisions (Springer, 1987).

17. J.L.S. Lino. cross-sections for electron-impact excitation of neutral atoms. Rev. Mex. Fis. 63, 190 (2017).

18. M.F. Gu. FAC 1.0.7. https://www-amdis.iaea.org/FAC/.

19. M.F. Gu. The flexible atomic code. Can. J. Phys. 86, 675 (2008).

20. D.L. Moores, H. Nussbaumer. The ionization of $\mathrm{Li}^{+}$and $\mathrm{Mg}^{+}$by electron impact. J. Phys. B: At. Mol. Phys. 3, 161 (1970).

21. M. Mattioli, G. Mazzitelli, M. Finkenthal, P. Mazzotta, K.B. Fournier, J. Kaastra, M.E .Puiatti. Updating of ionization data for ionization balance evaluations of atoms and ions for the elements hydrogen to germanium. J. Phys. B: At. Mol. Opt. Phys. 40, 3569 (2007).

22. M.S. Pindzola, D.M. Mitnik, J. Colgan, D.C. Griffin. Electron-impact ionization of $\mathrm{Li}^{+}$Phys. Rev. A 61, 052712 (2000).

23. A. Muller, G. Hofmann, B. Weissbecker, M. Stenke, K. Tinschert, M. Wagner, E. Salzborn. Correlated twoelectron transitions in electron-impact ionization of $\mathrm{Li}^{+}$ ions. Phys. Rev. Lett. 63, 758 (1989).

24. B. Peart, K.T. Dolder. Measurements of cross-sections for the ionization of $\mathrm{Li}^{+}$and $\mathrm{Ba}^{+}$ions by electron impact. J. Phys. B: At. Mol. Phys. 1, 872 (1968).
25. J.B. Wareing, K.T. Dolder. A measurement of the crosssection for ionization of $\mathrm{Li}^{+}$to $\mathrm{Li}^{2+}$ by electron impact. Proc. Phys. Soc. 91, 887 (1967).

26. W.C. Lineberger, J.W. Hooper, E.W. McDaniel. Absolute cross-sections for single ionization of alkali ions by electron impact. I. Description of Apparatus and Li ${ }^{+}$Results. Phys. Rev. 141, 151 (1966).

27. A. Borovik Jr, A. Muller, S. Schippers, I. Bray, D.V. Fursa. Electron impact ionization of ground-state and metastable $\mathrm{Li}^{+}$ions. J. Phys. B: At. Mol. Opt. Phys. 42, 025203 (2009).

28. S.B. Hansen, A.S. Shlyaptseva. Effects of the electron energy distribution function on modeled x-ray spectra. Phys. Rev. E 70, 036402 (2004).

29. A. Escarguel, F.B. Rosmej, C. Brault, T.H. Pierre, R. Stamm, K. Quotb. Influence of hot electrons on radiative properties of a helium plasma. Plasma Phys. Control. Fusion 49, 85 (2007).

Received 13.08.20

С. Ділмі, А. Бумалі

\section{ОЦІНКА ШВИДКОСТІ ЙОНІЗАЦІї $\mathrm{Li}$}

\section{ЕЛЕКТРОННИМ УДАРОМ 3 ВИКОРИСТАННЯМ} НЕМАКСВЕЛЛІВСЬКОЇ ФУНКЦІЇ РОЗПОДІЛУ

Зроблено оцінку поперечного перерізу і швидкості йонізації $\mathrm{Li}$ електронним ударом. Використано програмний код FAC (Flexible Atomic Code) для розрахунку поперечного перерізу і рівнів енергії. Розглянуто вплив немаксвеллівського розподілу по енергії на швидкість йонізації у випадку, коли частка гарячих електронів мала. Для деяких видів плазми було показано, що гарячі електрони мають немаксвеллівський розподіл по енергії і впливають на лінійний спектр і інші характеристики плазми. Ми також виявили чутливість швидкості йонізації Li електронним ударом до розподілу електронів по енергії і до частки гарячих електронів.

$K л$ ю ов $i$ cлов $а$ : код $\mathrm{FAC,} \mathrm{поперечний} \mathrm{переріз} \mathrm{йонізації,}$ функція розподілу, швидкість йонізації, немаксвеллівський розподіл. 